\title{
Global existence of classical solutions to the Vlasov-Poisson system in a three dimensional, cosmological setting
}

\author{
Gerhard Rein \\ Mathematisches Institut der Universität München \\ Theresienstr. 39, W8000 München 2, Germany \\ and \\ Alan D. Rendall \\ Max-Planck-Institut für Astrophysik \\ Karl-Schwarzschild-Str. 1, W8046 Garching, Germany
}

\begin{abstract}
The initial value problem for the Vlasov-Poisson system is by now well understood in the case of an isolated system where, by definition, the distribution function of the particles as well as the gravitational potential vanish at spatial infinity. Here we start with homogeneous solutions, which have a spatially constant, non-zero mass density and which describe the mass distribution in a Newtonian model of the universe. These homogeneous states can be constructed explicitly, and we consider deviations from such homogeneous states, which then satisfy a modified version of the Vlasov-Poisson system. We prove global existence and uniqueness of classical solutions to the corresponding initial value problem for initial data which represent spatially periodic deviations from homogeneous states.
\end{abstract}

\section{Introduction}

Consider a large ensemble of particles which interact only by the gravitational potential which they generate collectively. If the particles are described by a time-dependent distribution function $f=f(t, x, v)$ on phase space and if we neglect relativistic as well as collisional effects, then the time evolution of the ensemble is governed by the Vlasov-Poisson system

$$
\partial_{t} f+v \cdot \partial_{x} f-\partial_{x} U \cdot \partial_{v} f=0,
$$




$$
\triangle U=4 \pi \rho
$$

where

$$
\rho(t, x):=\int_{\mathbb{R}^{3}} f(t, x, v) d v
$$

denotes the spatial mass density generated by $f, U=U(t, x)$ denotes the gravitational potential, and $t \geq 0$ denotes time, $x \in \mathbb{R}^{3}$ position, and $v \in \mathbb{R}^{3}$ velocity. The same system - with a minus in front of the right hand side of Poisson's equation - models a plasma of electrons or ions which interact by electrostatic Coulomb forces. Here we are concerned only with the stellar dynamic case.

In the corresponding initial value problem one prescribes the phase space density at $t=0$, i. e. $f(0)=\stackrel{\circ}{ }$ is a given, nonnegative function on $\mathbb{R}^{6}$. However, stated in this form, this initial value problem is ill-posed. It has to be supplemented with a boundary condition for the potential $U$ at spatial infinity. If one wants to model an isolated system, for example a galaxy in an otherwise empty universe, one requires that

$$
\lim _{x \rightarrow \infty} U(t, x)=0, t \geq 0
$$

This boundary condition - together with some sort of decay of $\stackrel{\circ}{f}$ at infinity is used in all mathematical investigations of the Vlasov-Poisson system and related systems that we are aware of, with the exception of systems on bounded domains, considered in the plasma physics case.

In the context of general relativity it is traditional to consider also cosmological solutions. An isolated system can be thought of as a more or less localized deviation from flat space, whereas a cosmological solution is a deviation from a homogeneous state, usually one with non-zero, spatially constant mass density. The present paper investigates this situation in a nonrelativistic, Newtonian setting. Our main result is global existence and uniqueness of classical solutions for initial data which represent spatially periodic deviations from the homogeneous state. For the applications in cosmology see [9]. Note that very little is known about the existence of Newtonian cosmological models without symmetry. The only available result is a local in time existence theorem for the Euler-Poisson system [4]. The present paper proceeds as follows: In the next section we briefly describe spatially homogeneous solutions of the Vlasov-Poisson system where $f$ is locally isotropic in $v$. There are essentially two types of such solutions: solutions which exist for all future times and are expanding in a certain sense, and solutions which first expand, then contract, and collapse to a 
singular state in finite time. In general relativity, these would correspond to an "open" or "closed" universe respectively. Next we derive the VlasovPoisson-type system which holds for a deviation from such a homogeneous state. In Sect. 3 we fix some notation. A local existence and uniqueness result for classical solutions to the initial value problem is established in Sect. 4, together with a continuation criterion for such solutions. An important ingredient in the proof of global existence is a bound on the kinetic energy of the system. Such a bound will be established in Sect. 5. In the last section we finally prove that the solutions exist globally in time. In the case of an open universe, where the homogeneous background state exists for all future times, the meaning of this statement is clear, and, in addition, we obtain information on the asymptotic growth of the deviation. In the case of a closed universe, where the homogeneous state exists only for a finite time, "global" means that the deviation exists as long as the homogeneous state. This should not be mistaken for a merely local existence result: it might have been thought that the deviation develops a singularity before the final "big crunch" of the homogeneous state, but our result shows that this is not so.

To conclude this introduction we give some references to the literature where we restrict ourselves to investigations of the existence of global, classical solutions. In the case of an isolated system, global existence of classical solutions for the Vlasov-Poisson system with general initial data was first obtained by Pfaffelmoser [10]. His proof was greatly simplified and his asymptotic estimates improved by Schaeffer [13] and Horst [7], see also [3] for a spatially periodic version of these results in the plasma physics case. With completely different methods, Lions and Perthame [8] also obtained global existence for the Vlasov-Poisson system. A version of this system incorporating special relativistic effects was considered by Glassey and Schaeffer who showed that solutions of the relativistic Vlasov-Poisson system develop singularities in finite time in the stellar dynamic case if the initial data have negative energy [5]. However, this system has the defect that it is neither Galilei nor Lorentz invariant. A fully Lorentz invariant system, considered in the plasma physics case, is the relativistic Vlasov-Maxwell system, where the question of global existence of classical solutions for general data is open. We refer to [1] and the references therein for global results for special data. A fully relativistic version with gravitational interaction, the Vlasov-Einstein system, was investigated only recently in [12], and global existence was obtained for small, spherically symmetric data. As mentioned earlier, all these results treat the case of an isolated system. 


\section{Homogeneous solutions and the system for de- viations from these}

In this section we first investigate a class of solutions to the Vlasov-Poisson system (1.1), (1.2), (1.3) which are homogeneous in $x$ and locally isotropic in $v$. Then we shall derive a system of equations which is satisfied by deviations from such a homogeneous state.

For a nonnegative function $H \in C_{c}^{1}(\mathbb{R})$ we set

$$
f_{0}(t, x, v):=H\left(a^{2}(t)\left|v-\frac{\dot{a}(t)}{a(t)} x\right|^{2}\right)
$$

where $a$ is a positive, scalar function to be determined later. We obtain

$$
\rho_{0}(t, x)=\int f_{0}(t, x, v) d v=a^{-3}(t) \int H\left(v^{2}\right) d v,
$$

and, after normalizing

$$
\int H\left(v^{2}\right) d v=1
$$

we have the homogeneous mass density

$$
\rho_{0}(t)=a^{-3}(t), t \geq 0 .
$$

A solution of the corresponding Poisson equation is then given by

$$
U_{0}(t, x):=\frac{2 \pi}{3} a^{-3}(t) x^{2}, t \geq 0, x \in \mathbb{R}^{3} .
$$

This potential does not satisfy the boundary condition (1.4), but this cannot be expected from a solution of the Poisson equation (1.2) with a spatially constant, non-zero density. We note that

$$
\partial_{x} U_{0}(t, x)=\frac{4 \pi}{3} a^{-3}(t) x,
$$

and it remains to determine the function $a$ in such a way that $f_{0}$ satisfies the Vlasov equation with force term $-\partial_{x} U_{0}$. This is the case if the quantity

$$
a^{2}(t)\left|v-\frac{\dot{a}(t)}{a(t)} x\right|^{2}
$$


satisfies this equation. A short computation shows that

$$
\begin{aligned}
& \left(\partial_{t}+v \cdot \partial_{x}-\partial_{x} U_{0} \cdot \partial_{v}\right) a^{2}(t)\left|v-\frac{\dot{a}(t)}{a(t)} x\right|^{2}= \\
& -2 a(t)\left(v-\frac{\dot{a}(t)}{a(t)} x\right) \cdot x\left(\ddot{a}(t)+\frac{4 \pi}{3} a^{-2}(t)\right)
\end{aligned}
$$

which is zero for $t \geq 0, x, v \in \mathbb{R}^{3}$ if and only if $a$ is a solution of the differential equation

$$
\ddot{a}+\frac{4 \pi}{3} a^{-2}=0 .
$$

This equation describes radial motion in the gravitational field of a point mass, and it is well known and obvious that the quantity

$$
E_{a}:=\frac{1}{2} \dot{a}(t)^{2}-\frac{4 \pi}{3} a^{-1}(t)
$$

is conserved along solutions. Let $a:\left[0, T_{a}[\rightarrow] 0, \infty[\right.$ be a solution of Eqn. (2.4) on its right maximal interval of existence with $a(0)=1$. We can distinguish the following two cases, depending on whether the energy $E_{a}$ is positive or negative:

Case A, $\dot{a}(0) \geq \sqrt{8 \pi / 3}$ : The solution of Eqn. (2.4) exists on $[0, \infty[$ and $\dot{a}(t)>0$ for $t \geq 0$, i. e. the solution expands forever.

Case $\mathbf{B}, \dot{a}(0)<\sqrt{8 \pi / 3}$ : The maximal existence time $T_{a}$ is finite, $\lim _{t \rightarrow T_{a}} a(t)=0$, and there exists a time $t_{a} \in\left[0, T_{a}[\right.$ such that $\dot{a}(t)>0$ for $t \in\left[0, t_{a}[\right.$ and $\dot{a}(t)<0$ for $t \in] t_{a}, T_{a}$ [, i. e. the solution expands up to the time $t_{a}$ and then contracts and collapses to a singular state in finite time.

We remark that in both cases the function $a$ can be extended into the past only for a finite time, after which it collapses to a singular state, the "big bang".

We will now formulate the system of equations which is satisfied by perturbations of the homogeneous state $f_{0}, \rho_{0}, U_{0}$. Let

$$
f=f_{0}+g, \rho=\rho_{0}+\sigma, U=U_{0}+W
$$

be a solution of the Vlasov-Poisson system (1.1), (1.2), (1.3). A short computation shows that this is the case if and only if $g, \sigma, W$ solves the system

$$
\partial_{t} g+v \cdot \partial_{x} g-\left(\partial_{x} W+\frac{4 \pi}{3} a^{-3}(t) x\right) \cdot \partial_{v} g=\partial_{x} W \cdot \partial_{v} f_{0},
$$




$$
\begin{gathered}
\triangle W=4 \pi \sigma, \\
\sigma(t, x):=\int g(t, x, v) d v .
\end{gathered}
$$

Essentially, one can now consider two types of such deviations: One can consider deviations from the homogeneous background which are in some sense localized, say, the support of $g$ is compact initially, or one can consider deviations which extend over the whole universe in some periodic way. However, due to the non-zero right hand side in Eqn. (2.5) an initially compact support of the perturbation $g$ is not preserved by the time evolution of the system (2.5), (2.6), (2.7). Also, it seems physically more convincing to have the deviation from the homogeneous state extend over the whole universe instead of only some part of it. The explicit $x$-dependence of the coefficient in Eqn. (2.5) seems to preclude the possibility of periodic solutions to this system but this can be overcome by the following transformation of variables:

$$
\begin{aligned}
& \tilde{x}=a^{-1}(t) x \\
& \tilde{v}=v-a^{-1}(t) \dot{a}(t) x
\end{aligned} \quad \text { i. e. } \quad \begin{aligned}
& x=a(t) \tilde{x} \\
& v=\tilde{v}+\dot{a}(t) \tilde{x}
\end{aligned},
$$

and

$$
\begin{gathered}
\tilde{g}(t, \tilde{x}, \tilde{v})=g(t, x, v)=g(t, a(t) \tilde{x}, \tilde{v}+\dot{a}(t) \tilde{x}), \\
\tilde{\sigma}(t, \tilde{x})=\sigma(t, x)=\sigma(t, a(t) \tilde{x}), \\
\tilde{W}(t, \tilde{x})=W(t, x)=W(t, a(t) \tilde{x}) .
\end{gathered}
$$

Then $\tilde{g}, \tilde{\sigma}, \tilde{W}$ satisfy the system

$$
\begin{gathered}
\partial_{t} \tilde{g}+\frac{1}{a} \tilde{v} \cdot \partial_{\tilde{x}} \tilde{g}-\frac{1}{a}\left(\partial_{\tilde{x}} \tilde{W}+\dot{a} \tilde{v}\right) \cdot \partial_{\tilde{v}} \tilde{g}=2 a H^{\prime}\left(a^{2} \tilde{v}^{2}\right) \tilde{v} \cdot \partial_{\tilde{x}} \tilde{W} \\
\triangle \tilde{W}=4 \pi a^{2} \tilde{\sigma} \\
\tilde{\sigma}(t, \tilde{x}):=\int \tilde{g}(t, \tilde{x}, \tilde{v}) d \tilde{v} .
\end{gathered}
$$

Since throughout the present paper we will be concerned with this system in the transformed variables, we now drop the tilde and obtain the following system, describing the time evolution of a deviation from the homogeneous, locally isotropic state:

$$
\begin{gathered}
\partial_{t} g+\frac{1}{a} v \cdot \partial_{x} g-\frac{1}{a}\left(\partial_{x} W+\dot{a} v\right) \cdot \partial_{v} g=2 a H^{\prime}\left(a^{2} v^{2}\right) v \cdot \partial_{x} W \\
\triangle W=4 \pi a^{2} \sigma
\end{gathered}
$$




$$
\sigma(t, x):=\int g(t, x, v) d v
$$

where it should be kept in mind that $t \geq 0, x, v \in \mathbb{R}^{3}$ are related to the original variables by the transformation (2.8). We shall be interested in solutions of this system which are periodic in $x$; note that the coefficients in Eqn. (2.12) no longer depend on $x$ explicitly. It should be noted that the resulting solutions to the original Vlasov-Poisson system are not spatially periodic, since we have rescaled the space variable in a time-dependent manner; the change in the velocity variable simply means that the bulk velocity $a^{-1}(t) \dot{a}(t) x$ of the homogeneous background solution is shifted into the origin of the velocity space.

To conclude this section, we transform the original Vlasov-Poisson system (1.1), (1.2), (1.3) according to (2.8), drop the tilde and obtain:

$$
\begin{gathered}
\partial_{t} f+\frac{1}{a} v \cdot \partial_{x} f-\frac{1}{a}\left(\partial_{x} U-\frac{4 \pi}{3} a^{-1} x+\dot{a} v\right) \cdot \partial_{v} f=0, \\
\triangle U=4 \pi a^{2} \rho, \\
\rho(t, x):=\int f(t, x, v) d v .
\end{gathered}
$$

Throughout the paper we make the following assumption:

(A) $\left\{\begin{array}{l}a:\left[0, T_{a}[\rightarrow] 0, \infty[\text { is a maximal solution of Eqn. } 2.4) \text { with } a(0)=1\right. \\ H \in C_{c}^{1}(\mathbb{R}), H \geq 0, \int H\left(v^{2}\right) d v=1, \text { and } H\left(v^{2}\right)=0,|v| \geq u_{0}>0\end{array}\right.$

We define $f_{0}, \rho_{0}, U_{0}$ by

$$
f_{0}(t, x, v):=H\left(a^{2}(t) v^{2}\right), \rho_{0}(t):=a^{-3}(t), U_{0}(t, x):=\frac{2 \pi}{3} a^{-1}(t) x^{2},
$$

which are the equations (2.1), (2.2), (2.3), transformed according to (2.8). Then $f_{0}, \rho_{0}, U_{0}$ is a solution of the transformed Vlasov-Poisson system (2.15), (2.16), 2.17), which we refer to as the homogeneous, locally isotropic background state of the Vlasov-Poisson system. If $g, \sigma, W$ satisfy the system (2.12), (2.13), (2.14) then $f=f_{0}+g, \rho=\rho_{0}+\sigma, U=U_{0}+W$ is a solution of (2.15), (2.16), (2.17), and, via the transformation (2.8), also a solution of the original Vlasov-Poisson system (1.1), (1.2), (1.3), which we refer to as a cosmological solution of the Vlasov-Poisson system, and which no longer has to be homogeneous or isotropic. 


\section{Some notation}

Let $Q:=[0,1]^{3}$ and $S:=Q \times \mathbb{R}^{3}$. We need to define some spaces of periodic functions:

$$
\begin{aligned}
\mathcal{P}(Q) & :=\left\{h: \mathbb{R}^{3} \rightarrow \mathbb{R} \mid h(x+\alpha)=h(x), x \in \mathbb{R}^{3}, \alpha \in \mathbb{Z}^{3}\right\}, \\
\mathcal{P}(S) & :=\left\{h: \mathbb{R}^{6} \rightarrow \mathbb{R} \mid h(x+\alpha, v)=h(x, v), x, v \in \mathbb{R}^{3}, \alpha \in \mathbb{Z}^{3}\right\}, \\
C_{\pi}^{n}(Q) & :=C^{n}(Q) \cap \mathcal{P}(Q), \\
C_{\pi}^{n}(S) & :=C^{n}(S) \cap \mathcal{P}(S), \\
C_{\pi, c}^{n}(S) & :=\left\{h \in C_{\pi}^{n}(S)|\exists u \geq 0: h(x, v)=0,| v \mid>u\right\} .
\end{aligned}
$$

For $p \in[1, \infty]$ we denote by $\|\cdot\|_{p}$ the usual $L^{p}$-norm, where the integral (or supremum) extends over $Q$ or $S$ as the case may be, and

$$
\begin{aligned}
L_{\pi}^{p}(Q) & :=L^{p}(Q) \cap \mathcal{P}(Q), \\
L_{\pi}^{p}(S) & :=L^{p}(S) \cap \mathcal{P}(S),
\end{aligned}
$$

where we identify functions in $\mathcal{P}(Q)$ and $\mathcal{P}(S)$ with their restrictions to $Q$ or $S$ respectively. For the pair $(x, v)$ we frequently write $z$.

\section{Local existence and continuation of solutions}

In this section we shall prove the existence of local solutions of the system (2.12), (2.13), (2.14) for initial data in $C_{\pi, c}^{1}(S)$. To do this, we have to be able to solve the equations (2.12) and (2.13) seperately. We start by investigating the Poisson equation for a given, spatially periodic density.

Lemma 4.1 There exists an even function $G \in C^{\infty}\left(\mathbb{R}^{3} \backslash \mathbb{Z}^{3}\right) \cap \mathcal{P}(Q)$ with the following properties:

(a) For $\sigma \in C_{\pi}^{1}(Q)$ with $\int_{Q} \sigma(x) d x=0$ the function

$$
W(x):=\int_{Q} G(x-y) \sigma(y) d x=\int_{x^{\prime}+Q} G(x-y) \sigma(y) d y, x, x^{\prime} \in \mathbb{R}^{3}
$$

is in $C_{\pi}^{2}(Q)$ the unique solution of

$$
\triangle W=4 \pi \sigma
$$

with $\int_{Q} W(x) d x=0$. 
(b) There exists a function $G_{0} \in C^{\infty}\left(\left(\mathbb{R}^{3} \backslash \mathbb{Z}^{3}\right) \cup\{(0,0,0)\}\right)$ such that

$$
G(x)=-\frac{1}{|x|}+G_{0}(x), x \in \mathbb{R}^{3} \backslash \mathbb{Z}^{3} .
$$

For a proof of this result we refer to [2]. Next we note some estimates for the potential $W$ which can be proved by treating the singular term in the Green's function $G$ as in [1, Props. 1, 2].

Lemma 4.2 There exists a constant $C>0$ such that for all $\sigma$ as in Lemma 4.1 the corresponding potential $W$ satisfies the following estimates:

(a) $\|W\|_{\infty} \leq C\left(\|\sigma\|_{1}^{2 / 3}\|\sigma\|_{\infty}^{1 / 3}+\|\sigma\|_{1}\right)$

(b) $\left\|\partial_{x} W\right\|_{\infty} \leq C\left(\|\sigma\|_{1}^{1 / 3}\|\sigma\|_{\infty}^{2 / 3}+\|\sigma\|_{1}\right)$

(c) $\left\|\partial_{x}^{2} W\right\|_{\infty} \leq C\left(\left(1+R^{-3}\right)\|\sigma\|_{1}+(1+\ln R / d)\|\sigma\|_{\infty}+d\left\|\partial_{x} \sigma\right\|_{\infty}\right)$ for any $d, R$ with $0<d \leq R \leq 1 / 2$

(d) $\left\|\partial_{x}^{2} W\right\|_{\infty} \leq C\left(\|\sigma\|_{1}+\left(1+\|\sigma\|_{\infty}\right)\left(1+\ln ^{*}\left\|\partial_{x} \sigma\right\|_{\infty} / 2\right)\right)$, where

$$
\ln ^{*} s:=\left\{\begin{array}{cc}
s & , s \leq 1 \\
1+\ln s & , s>1
\end{array},\right.
$$

(e) $\left\|\partial_{x} W\right\|_{\infty} \leq C\left(\|\sigma\|_{5 / 3}^{5 / 9}\|\sigma\|_{\infty}^{4 / 9}+\|\sigma\|_{1}\right)$.

Next we investigate Eqn. (2.12) for a given potential $W$. To this end, we first consider the corresponding characteristic system

$$
\dot{x}=\frac{1}{a(t)} v, \dot{v}=-\frac{1}{a(t)}\left(\partial_{x} W(t, x)+\dot{a}(t) v\right) .
$$

Lemma 4.3 Let $0<T \leq T_{a}$ and $W \in C_{\pi}^{0,2}([0, T[\times Q)$. Then the following holds:

(a) For $z=(x, v) \in \mathbb{R}^{6}$ and $t \in[0, T[$ there exists a unique solution

$$
[0, T[\ni s \mapsto(X(s, t, x, v), V(s, t, x, v))=Z(s, t, z)
$$

of the characteristic system (4.1) with $Z(t, t, z)=z$. 
(b) $Z \in C^{1}\left(\left[0, T\left[^{2} \times \mathbb{R}^{6}\right)\right.\right.$, for every $s, t \in\left[0, T\left[\right.\right.$ the mapping $Z(s, t, \cdot)$ is a $C^{1}$ diffeomorphism of $\mathbb{R}^{6}$, and

$$
Z(s, t, x+\alpha, v)=Z(s, t, x, v)+(\alpha, 0), \alpha \in \mathbb{Z}^{3} .
$$

(c) $\operatorname{det} \partial_{z} Z(s, t, z)=\left(\frac{a(t)}{a(s)}\right)^{3}, s, t \in[0, T[$.

Proof : Part (a) and the first two assertions in (b) are standard, the third assertion in (b) follows by uniqueness and the periodicity of $W$. For (c), note that

$$
\operatorname{div}_{z}\left(\frac{1}{a} v,-\frac{1}{a}\left(\partial_{x} W+\dot{a} v\right)\right)=-3 \frac{\dot{a}}{a} .
$$

Thus

$$
\frac{d}{d s}\left(\operatorname{det} \partial_{z} Z(s, t, z)\right)=-3 \frac{\dot{a}(s)}{a(s)} \operatorname{det} \partial_{z} Z(s, t, z),
$$

and the result follows.

Lemma 4.4 Let $W$ satisfy the assumption in Lemma 4.3 and take $\stackrel{\circ}{g} \in$ $C_{\pi, c}^{1}(S)$ such that $\stackrel{\circ}{g}(z)+H\left(v^{2}\right) \geq 0$ for $z \in \mathbb{R}^{6}$. Then

$$
\begin{aligned}
g(t, z) & :=\stackrel{\circ}{g}(Z(0, t, z))+2 \int_{0}^{t} a(s)\left(H^{\prime}\left(a^{2} v^{2}\right) v \cdot \partial_{x} W\right)(s, Z(s, t, z)) d s \\
& =\stackrel{\circ}{g}(Z(0, t, z))+H\left(V^{2}(0, t, z)\right)-H\left(a^{2}(t) v^{2}\right), t \in\left[0, T\left[, \quad z \in \mathbb{R}^{6}\right.\right.
\end{aligned}
$$

defines the unique solution of Eqn. (2.19) in $C^{1}\left(\left[0, T\left[\times \mathbb{R}^{6}\right)\right.\right.$ with $g(0)=\stackrel{g}{g}$. The solution $g$ has the following properties:

(a) $g \in C\left(\left[0, T\left[; C_{\pi, c}^{1}(S)\right)\right.\right.$,

(b) $\int_{S} \stackrel{\circ}{g}(z) d z=0$ implies $\int_{S} g(t, z) d z=0, t \in[0, T[$,

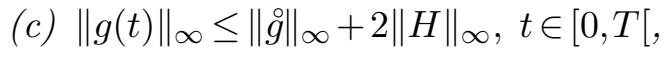

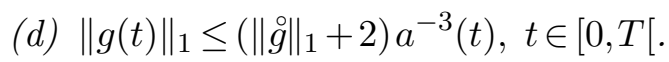

Proof : The fact that Eqn. (4.2) defines the unique $C^{1}$-solution of Eqn. (2.12) with $g(0)=\stackrel{g}{g}$ is standard if $H \in C^{2}(\mathbb{R})$. Since $-H\left(a^{2}(s) V^{2}(s, t, z)\right)$ is a primitive of the integrand in (4.2), Eqn. (4.3) defines the same function, and an approximation argument for $H$ shows that it is the unique $C^{1}$-solution also if $H \in C^{1}(\mathbb{R})$. The assertion in (a) follows from the periodicity of $\stackrel{\circ}{g}$ and 
Lemma 4.3. To see the assertion in (b), integrate Eqn. 2.12) with respect to $z$, observe that the right hand side in that equation is odd in $v$, and integrate by parts with respect to $x$ and $v$ respectively to obtain the equation

$$
\frac{d}{d t} \int_{S} g(t, z) d z=-3 \frac{\dot{a}(t)}{a(t)} \int_{S} g(t, z) d z, t \in[0, T[;
$$

observe that no boundary terms occur due to the compact support in $v$ and the periodicity in $x$ respectively. The assertion in (b) follows but observe that we obtain no information on $\|g(t)\|_{1}$ from this since $g$ is not positive. The assertion in (c) is obvious by (4.3). To prove (d), define $f:=f_{0}+g$. Then $f$ satisfies the equation

$$
\partial_{t} f+\frac{1}{a} v \cdot \partial_{x} f-\frac{1}{a}\left(\partial_{x} W+\dot{a} v\right) \cdot \partial_{v} f=0
$$

so that

$$
f(t, z)=f(0, Z(0, t, z))=H\left(V^{2}(0, t, z)\right)+\stackrel{\circ}{g}(Z(0, t, z)) \geq 0
$$

by assumption on $\stackrel{\circ}{g}$, and $f(t) \in C_{\pi}^{1}(S), t \in[0, T[$. The same argument as above proves that

$$
\frac{d}{d t} \int_{S} f(t, z) d z=-3 \frac{\dot{a}(t)}{a(t)} \int_{S} f(t, z) d z, t \in[0, T[.
$$

Thus

$$
\|f(t)\|_{1}=\|f(0)\|_{1} a^{-3}(t)
$$

and since

$$
\left\|f_{0}(t)\right\|_{1}=a^{-3}(t)
$$

the assertion in $(\mathrm{d})$ follows.

We are now ready to prove local existence of classical solutions to the initial value problem for the system (2.12), (2.13), 2.14).

Theorem 4.5 Let $\stackrel{\circ}{g} \in C_{\pi, c}^{1}(S)$ with $\int_{S} \stackrel{\circ}{g}(z) d z=0$ and $\stackrel{\circ}{g}(x, v)+H\left(v^{2}\right) \geq$ $0,(x, v) \in \mathbb{R}^{6}$. Then there exists a unique maximal solution

$$
g \in C^{1}\left(\left[0, T\left[\times \mathbb{R}^{6}\right) \cap C\left(\left[0, T\left[; C_{\pi, c}^{1}(S)\right)\right.\right.\right.\right.
$$

of the system (2.19), 2.19), 2.14) with $g(0)=\stackrel{9}{g}, 0<T \leq T_{a}$. Moreover, if

$$
\sup \{|v| \mid(x, v) \in \operatorname{supp} g(t), 0 \leq t<T\}<\infty
$$

then $T=T_{a}$, $i$. e. the solution exists as long as the homogeneous state. 
Proof : The method of proof is quite standard, and we only indicate the main steps; for an isolated system the corresponding arguments can be found in [1].

Consider the following iterative scheme: Define

$$
g_{0}(t, z):=\stackrel{\circ}{g}(z), t \in\left[0, T_{a}\left[, z \in \mathbb{R}^{6},\right.\right.
$$

and if

$$
g_{n} \in C^{1}\left(\left[0, T\left[\times \mathbb{R}^{6}\right) \cap C\left(\left[0, T\left[; C_{\pi, c}^{1}(S)\right)\right.\right.\right.\right.
$$

is already defined,

$$
\begin{gathered}
\sigma_{n}(t, x):=\int g_{n}(t, x, v) d v \\
W_{n}(t, x):=a^{2}(t) \int_{Q} G(x-y) \sigma_{n}(t, y) d y
\end{gathered}
$$

for $t \in\left[0, T_{a}\left[, x \in \mathbb{R}^{3}\right.\right.$, let $Z_{n+1}$ be defined according to Lemma 4.3 with $W_{n}$ instead of $W$, and

$g_{n+1}(t, z):=\stackrel{\circ}{g}\left(Z_{n+1}(0, t, z)\right)+H\left(V_{n+1}^{2}(0, t, z)\right)-H\left(a^{2}(t) v^{2}\right), t \in\left[0, T_{a}\left[, z \in \mathbb{R}^{6}\right.\right.$.

It follows from the above Lemmata that this scheme is well defined. With

$$
\begin{aligned}
P_{n}(t):= & \sup \left\{\left|V_{n-1}(s, 0, z)\right| \mid z \in \operatorname{supp} g \cup \mathbb{R}^{3} \times B_{u_{0}}(0), 0 \leq s \leq t\right\} \\
& +u_{0} a^{-1}(t), t \in\left[0, T_{a}[,\right.
\end{aligned}
$$

it follows that

$$
g_{n}(t, x, v)=0,|v|>P_{n}(t) .
$$

Using the characteristic system and the estimates from Lemma 4.2 and Lemma 4.4 it can be shown by induction that

$$
P_{n}(t) \leq P(t), t \in[0, \delta[, n \in \mathbb{N}
$$

where $P:\left[0, \delta\left[\rightarrow \mathbb{R}^{+}\right.\right.$is the right maximal solution of the equation

$$
P(t)=P_{0}+C(\stackrel{\circ}{g}) \int_{0}^{t}\left(P^{2}(s)+\frac{|\dot{a}(s)|}{a(s)} P(s)+a^{-2}(s)\right) d s+u_{0} a^{-1}(t),
$$

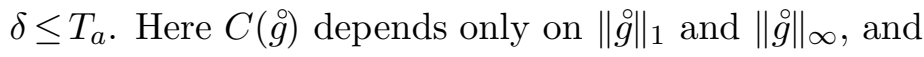

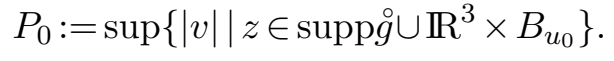


On the interval $[0, \delta[$ the iterative scheme can now be seen to converge. First observe that

$$
\left\|\sigma_{n}(t)\right\|_{\infty} \leq C P^{3}(t),\left\|\partial_{x} W(t)\right\|_{\infty} \leq C\left(a^{-1}(t)+a(t) P^{2}(t)\right), n \in \mathbb{N}, t \in[0, \delta[.
$$

Next it can be shown that on any compact subinterval $\left[0, \delta_{0}\right]$ of $[0, \delta[$

$$
\left\|\partial_{x} \sigma_{n}(t)\right\|_{\infty} \leq C,\left\|\partial_{x}^{2} W(t)\right\|_{\infty} \leq C, n \in \mathbb{N} .
$$

This can be seen from the following Gronwall-type inequality

$$
\left\|\partial_{x} \sigma_{n}(t)\right\|_{\infty} \leq C \exp \left(\int_{0}^{t} \ln * \frac{\left\|\partial_{x} \sigma_{n-1}(s)\right\|_{\infty}}{2} d s\right)
$$

which follows from Lemma 4.2 (d). Now define

$$
\alpha_{n}(t):=\sup \left\{\left|X_{n+1}-X_{n}\right|(s, t, z)+\left|V_{n+1}-V_{n}\right|(s, t, z) \mid 0 \leq s \leq t, z \in \mathbb{R}^{6}\right\}
$$

for $t \in\left[0, \delta_{0}\right]$. Using the uniform bounds which hold on the interval $\left[0, \delta_{0}\right]$ another Gronwall argument shows that

$$
\alpha_{n}(t) \leq C \int_{0}^{t} \alpha_{n-1}(s) d s, 0 \leq t \leq \delta_{0}, n \geq 1 .
$$

Thus, the sequence $Z_{n}$ is a uniform Cauchy sequence on $\left[0, \delta_{0}\right]^{2} \times \mathbb{R}^{6}$. The convergence of $g_{n}(t), \sigma_{n}(t), W_{n}(t)$ in $C_{\pi}(S), C_{\pi}(Q)$, and $C_{\pi}^{1}(Q)$ respectively follows, uniformly on $\left[0, \delta_{0}\right]$. Using Lemma 4.2 (c) it can be seen that $W_{n}(t)$ is actually a Cauchy sequence in $C_{\pi}^{2}(Q)$, uniformly on $\left[0, \delta_{0}\right]$. Thus, the function $g$ is $C^{1}$, and since $\delta_{0}<\delta$ is arbitrary we obtain the desired classical solution on the interval $[0, \delta[$.

To obtain uniqueness, the Gronwall arguments indicated above may be used on the difference of two solutions with the same initial datum.

Let $g \in C^{1}\left(\left[0, T\left[\times \mathbb{R}^{6}\right)\right.\right.$ be the unique maximal solution, and assume that $T<T_{a}$ and

$$
P^{*}:=\sup \{|v| \mid(x, v) \in \operatorname{supp} g(t), 0 \leq t<T\}<\infty .
$$

Then by Lemma 4.4

$$
\|g(t)\|_{1}+\|g(t)\|_{\infty} \leq C, t \in[0, T[.
$$

Consider now Eqn. (4.4), but with $g\left(t_{0}\right)$ instead of $\stackrel{\circ}{g}$ and $P^{*}$ instead of $P_{0}$. Then the function $P$ exists on an interval $\left[t_{0}, t_{0}+\delta[\right.$, the length of which is bounded from below by a positive constant, which is independent of $t_{0} \in$ $\left[0, T\left[\right.\right.$. Moving $t_{0}$ close enough to $T$ the above proof shows that there exists a solution on the interval $\left[t_{0}, t_{0}+\delta[\right.$ which extends the solution $g$ beyond the time $T$. This is a contradiction, and the proof is complete. 


\section{An energy estimate}

Throughout the rest of this paper $(g, \sigma, W)$ denotes a solution of the system (2.12), (2.13), 2.14) on a maximal interval of existence $\left[0, T\left[\subset\left[0, T_{a}[\right.\right.\right.$ as obtained in Theorem 4.5. By $C$ we shall denote constants which may change from line to line and may depend on $\stackrel{g}{g}$ but never on $t$. We want to establish a bound on the kinetic energy which in turn shall give a bound on $\|g(t)\|_{5 / 3}$. This is desirable because it allows the force term $\partial_{x} W$ to be bounded by a lower power of $\|\sigma(t)\|_{\infty}$, cf. Lemma 4.2 (e). However, the latter argument immediately runs into the problem that $g$ is not necessarily positive, and a bound on the kinetic energy of $g$ is useless when trying to control $\|g(t)\|_{5 / 3}$. To avoid this problem, we consider $f:=f_{0}+g$ instead. This function satisfies the equation

$$
\partial_{t} f+\frac{1}{a} v \cdot \partial_{x} f-\frac{1}{a}\left(\partial_{x} W+\dot{a} v\right) \cdot \partial_{v} f=0 .
$$

Thus it is constant along characteristics, and since it is nonnegative initially by assumption on $\stackrel{\circ}{g}$, is nonnegative for all $t \in[0, T[$. Now define

$$
\begin{gathered}
E_{k i n}(t):=\int_{S} v^{2} f(t, z) d z \\
E_{p o t}(t):=\int_{Q} W(t, x) \sigma(t, x) d x=-\frac{1}{4 \pi a^{2}(t)} \int_{Q}\left|\partial_{x} W(t, x)\right|^{2} d x, t \in[0, T[.
\end{gathered}
$$

If we differentiate the kinetic energy with respect to time, use the differential equation for $f$, and integrate by parts we obtain

$$
\frac{d}{d t} E_{k i n}(t)=-5 \frac{\dot{a}(t)}{a(t)} E_{k i n}(t)+\frac{2}{a(t)} \int_{Q} W(t, x) \operatorname{div} j(t, x) d x,
$$

where

$$
j(t, x):=\int v g(t, x, v) d v
$$

$f_{0}$, being even in $v$, does not contribute to the mass current density. Integrating (2.12) with respect to $v$ yields

$$
\partial_{t} \sigma+\frac{1}{a} \operatorname{div} j+3 \frac{\dot{a}}{a} \sigma=0
$$

and inserting this into (5.1) we obtain

$$
\frac{d}{d t} E_{k i n}(t)=-5 \frac{\dot{a}(t)}{a(t)} E_{k i n}(t)-2 \int_{Q} W(t, x) \partial_{t} \sigma(t, x) d x-6 \frac{\dot{a}}{a} E_{p o t}(t) .
$$


Differentiating the potential energy yields

$$
\frac{d}{d t} E_{p o t}(t)=2 \int_{Q} W(t, x) \partial_{t} \sigma(t, x) d x+2 \frac{\dot{a}}{a} E_{p o t}(t) .
$$

From Eqns. (5.2) and (5.3) it follows that

$$
a^{5}(t) E(t)=E(0)+\int_{0}^{t} \dot{a}(s) a^{4}(s) E_{p o t}(s) d s, t \in[0, T[,
$$

where $E:=E_{k i n}+E_{\text {pot }}$ denotes the total energy. To exploit Eqn. (5.4) we need to estimate the potential energy in terms of the kinetic energy. This argument is due to Horst [6, (5.7)], and we only write down the result for our present situation:

Lemma 5.1 For $t \in[0, T[$,

$$
\left|E_{\text {pot }}(t)\right| \leq C\left(a^{-4}(t)+a^{-3 / 2}(t) E_{k i n}(t)^{1 / 2}\right) .
$$

To continue, it is convenient to distinguish between Case A and Case B. In Case A, $\dot{a}>0$, and since the potential energy is negative, Eqn. (5.4) implies that

$$
E(t) \leq a^{-5}(t) E(0) .
$$

With Lemma 5.1 this yields

$$
E_{k i n}(t) \leq C\left(a^{-4}(t)+a^{-3 / 2}(t) E_{k i n}^{1 / 2}(t)\right), t \in[0, T[,
$$

which gives a bound on the kinetic energy in Case A. In Case B where $\dot{a}$ eventually becomes negative, we have to argue differently. Observing that in this case $a$ is bounded and $T \leq T_{a}<\infty$ we obtain from (5.4) and Lemma 5.1 the estimate

$$
\begin{aligned}
a^{5}(t) E_{k i n}(t) \leq & C\left(1+\int_{0}^{t}|\dot{a}(s)| a^{4}(s)\left(a^{-4}(s)+a^{-3 / 2} E_{k i n}(s)\right)^{1 / 2}\right) d s \\
& \left.\left.+a(t)+a^{7 / 2}(t) E_{k i n}(t)\right)^{1 / 2}\right) \\
\leq & C\left(1+\max _{0 \leq s \leq t}\left(a^{5}(s) E_{k i n}(s)\right)^{1 / 2}\right)\left(1+\int_{0}^{t}|\dot{a}(s)| d s\right) \\
\leq & C\left(1+\max _{0 \leq s \leq t}\left(a^{5}(s) E_{k i n}(s)\right)^{1 / 2}\right)
\end{aligned}
$$

which yields a bound for the kinetic energy in Case B. Thus we have proved the following result: 
Lemma 5.2 For $t \in\left[0, T\left[, E_{k i n}(t) \leq C a^{-3}(t)\right.\right.$ in Case $A$, and $E_{k i n}(t) \leq$ $C a^{-5}(t)$ in Case B.

By an interpolation argument and estimating $g$ in terms of $f+f_{0}$, these estimates yield the following bounds on $\|\sigma(t)\|_{5 / 3}$ :

Corollary 5.3 For $t \in\left[0, T\left[, \quad\|\sigma(t)\|_{5 / 3} \leq C a^{-9 / 5}(t) \quad\right.\right.$ in Case $A$, and $\|\sigma(t)\|_{5 / 3} \leq C a^{-3}(t)$ in Case $B$.

Thus we see that although we have no energy conservation for our problem, the usual bounds on the kinetic energy and the consequences thereof still hold.

\section{Global existence}

In this section we prove the following global existence result which is the main result of the present paper:

Theorem 6.1 Let $\stackrel{\circ}{g} \in C_{c, \pi}^{1}(S)$ with $\int_{S} \stackrel{\circ}{g}(z) d z=0$ and $\stackrel{\circ}{g}(z)+H\left(v^{2}\right) \geq 0$ for $z \in \mathbb{R}^{6}$. Then there exists a unique solution $g \in C^{1}\left(\left[0, T_{a}\left[\times \mathbb{R}^{6}\right) \cap\right.\right.$ $C\left(\left[0, T_{a}\left[; C_{\pi}^{1}(S)\right)\right.\right.$ of the system (2.19), 2.13), (2.14) with $g(0)=\stackrel{\circ}{g}$. Moreover, in Case $A$, where $T_{a}=\infty$, we have the estimate

$$
P(t)=O\left(t^{2+\delta}\right), t \geq 0
$$

for any $\delta>0$ where

$$
P(t):=\sup \{|v| \mid z \in \operatorname{supp} g(s), 0 \leq s \leq t\} .
$$

Proof : The proof makes use of the ideas of Schaeffer [13] and some unpublished simplifications of these [14]. Similar estimates have also been used in [7]. We first consider Case A. Along any characteristic we have the equation

$$
\frac{d}{d s}(a(s) v(s))=\dot{a}(s) v(s)-\partial_{x} W(s, x(s))-\dot{a}(s) v(s)=-\partial_{x} W(s, x(s)) .
$$

By Lemma 4.2 (e) and Cor. 5.3

$$
\left\|\partial_{x} W(s)\right\|_{\infty} \leq C a^{2}(s)\left(a^{-1}(s)\|\sigma(s)\|_{\infty}^{4 / 9}+\|\sigma(s)\|_{1}\right) \leq C_{1} a(s) P(s)^{4 / 3}
$$


and thus

$$
\left|a\left(s_{1}\right) v\left(s_{1}\right)-a\left(s_{2}\right) v\left(s_{2}\right)\right| \leq C_{1} a(t) P(t)^{4 / 3}\left|s_{1}-s_{2}\right|, 0 \leq s_{1}, s_{2} \leq t .
$$

Let $(X, V)$ denote a fixed characteristic which hits the support of $g$, and take $t \in[0, T[$ and $\Delta \in[0, t], T$ being the length of the maximal existence interval of the local solution $g$ corresponding to the initial value $\stackrel{\circ}{g}$. Then

$$
\begin{aligned}
& |a(t) V(t)-a(t-\Delta) V(t-\Delta)| \\
& \leq \int_{t-\Delta}^{t} a^{2}(s)\left(\int_{X(s)+\hat{Q}} \int_{\mathbb{R}^{3}}|g(s, x, v)| d v \frac{d x}{|x-X(s)|^{2}}\right. \\
& \left.+\left\|\partial_{x} G_{0}\right\|_{\infty} \int_{X(s)+\hat{Q}} \int_{\mathbb{R}^{3}}|g(s, x, v)| d v d x\right) d s \\
& \leq C \int_{t-\Delta}^{t} a^{-1}(s) d s+C \int_{t-\Delta}^{t} a^{2}(s) \int_{X(s)+\hat{Q}} \int_{\mathbb{R}^{3}}|g(s, x, v)| d v \frac{d x}{|x-X(s)|^{2}} d s
\end{aligned}
$$

where $\hat{Q}:=[-1 / 2,1 / 2]^{3}$. We split the domain of integration of the second term into the following parts:

$$
\begin{aligned}
& M_{1}:=\{(s, x, v) \mid D(s, v) \leq p\}, \\
& M_{2}:=\left\{(s, x, v)|D(s, v)>p \wedge| x-X(s) \mid \leq r D(s, v)^{-3}\right\}, \\
& M_{3}:=\left\{(s, x, v)|D(s, v)>p \wedge| x-X(s) \mid>r D(s, v)^{-3}\right\} .
\end{aligned}
$$

Here $D(s, v):=\min \{|v|,|v-V(s)|\}$, and $r>0, p>0$ are parameters to be chosen later, $p \leq P(t)$. Let $I_{i}$ denote the contribution of the set $M_{i}$ to the above integral. The integral $I_{1}$ is easily estimated:

$$
I_{1} \leq \int_{t-\Delta}^{t} a^{2}(s) \int_{X(s)+\hat{Q}} \tilde{\sigma}(s, x) \frac{d x}{|x-X(s)|^{2}} d s
$$

where

$$
\tilde{\sigma}(s, x):=\int_{D(s, v) \leq p}|g(s, x, v)| d v
$$

Now

$$
\|\tilde{\sigma}(s)\|_{5 / 3} \leq C a^{-9 / 5}(s),\|\tilde{\sigma}(s)\|_{\infty} \leq C p^{3}
$$

so that by Lemma 4.2 (e)

$$
I_{1} \leq C p^{4 / 3} a(t) \Delta .
$$


The estimate $I_{2}$ is straightforward too:

$$
\begin{aligned}
I_{2} & \leq \int_{t-\Delta}^{t} a^{2}(s) \int_{p \leq D(s, v) \leq 2 P(s)} \int_{|x-X(s)| \leq r D(s, v)^{-3}}|g(s, x, v)| \frac{d x d v d s}{|x-X(s)|^{2}} \\
& \leq \int_{t-\Delta}^{t} a^{2}(s) \int_{p \leq D(s, v) \leq 2 P(s)} r D(s, v)^{-3} d v d s \\
& \leq C a^{2}(t) r \ln \frac{2 P(t)}{p} \Delta .
\end{aligned}
$$

To estimate $I_{3}$ we want to use the transformation of variables

$$
z^{\prime}=Z(t, s, z)
$$

but since $g$ is not constant along characteristics we switch to $f=f_{0}+g$ first and obtain the estimate

$$
\begin{aligned}
I_{3} \leq & \int_{t-\Delta}^{t} a^{2}(s) \int_{X(s)+\hat{Q}} \int_{\mathbb{R}^{3}}|f(s, x, v)| 1_{M_{3}}(s, z) d v \frac{d x}{|x-X(s)|^{2}} d s \\
& +\int_{t-\Delta}^{t} a^{2}(s) \int_{X(s)+\hat{Q}} \int_{\mathbb{R}^{3}}\left|f_{0}(s, x, v)\right| d v \frac{d x}{|x-X(s)|^{2}} d s \\
\leq & J+C \int_{t-\Delta}^{t} a^{-1}(s) d s
\end{aligned}
$$

where $J$ denotes the integral over $f$ which remains to be estimated. Let $\Delta \leq \min \left\{t, \frac{1}{2 \dot{a}(0)}\right\}$. Since $\dot{a}$ is positive and decreasing,

$$
\frac{1}{2} a\left(s_{2}\right) \leq a\left(s_{1}\right) \leq a\left(s_{2}\right), t-\Delta \leq s_{1} \leq s_{2} \leq t .
$$

Using this estimate and Lemma 4.3 in the transformation of variables introduced above we obtain the following estimate:

$$
J \leq C \int_{t-\Delta}^{t} a^{2}(s) \int_{Z\left(t, s, X(s)+\hat{Q}, \mathbb{R}^{3}\right)}|f(t, z)| 1_{M_{3}}(s, Z(s, t, z)) \frac{d z d s}{|X(s, t, z)-X(s)|^{2}} .
$$

Now observe that

$$
Z\left(t, s, X(s)+\hat{Q}, \mathbb{R}^{3}\right) \cap \operatorname{supp} f(t) \subset(X(t)+2 \hat{Q}) \times \mathbb{R}^{3}:=M(t)
$$

if we assume that $P(t) \Delta \leq 1 / 4$ i. e. $\Delta \leq \min \left\{t, \frac{1}{2 \dot{a}(0)}, \frac{1}{4 P(t)}\right\}$. Defining

$$
J(z):=\left\{s \in[t-\Delta, t] \mid(s, Z(s, t, z)) \in M_{3}\right\}
$$


we obtain the estimate

$$
J \leq C \int_{M(t)} f(t, z) \int_{J(z)} a^{2}(s)|X(s, t, z)-X(s)|^{-2} d s d z .
$$

To estimate the time integral we need to restrict the length of the integration interval so that velocities do not change very much during that interval. We have

$$
\begin{aligned}
\left|v\left(s_{1}\right)-v\left(s_{2}\right)\right| & \leq \frac{1}{a\left(s_{1}\right)}\left|a\left(s_{1}\right) v\left(s_{1}\right)-a\left(s_{2}\right) v\left(s_{2}\right)\right|+\frac{\left|a\left(s_{1}\right)-a\left(s_{2}\right)\right|}{a\left(s_{1}\right)}\left|v\left(s_{2}\right)\right| \\
& \leq C_{1} \frac{a(t)}{a\left(s_{1}\right)} P(t)^{4 / 3} \Delta+\frac{\dot{a}(0)}{a\left(s_{1}\right)} P(t) \Delta \\
& \leq C_{2} P(t)^{4 / 3} \Delta
\end{aligned}
$$

for $s_{1}, s_{2} \in[t-\Delta, t]$; without loss of generality we may assume $P(t) \geq 1$. Thus if we choose

$$
\Delta:=\min \left\{t, \frac{1}{2 \dot{a}(0)}, \frac{1}{4 P(t)}, \frac{p}{5 C_{2} P(t)^{4 / 3}}\right\}
$$

the estimate

$$
\left|v\left(s_{1}\right)-v\left(s_{2}\right)\right| \leq \frac{p}{5}
$$

holds for $s_{1}, s_{2} \in[t-\Delta, t]$ and any characteristic which hits suppg. Now take $z \in M(t)$ and assume $J(z) \neq \emptyset$. Then for $s \in[t-\Delta, t]$ and $\sigma \in J(z)$ the above estimate and the definition of the set $M_{3}$ imply

$$
\begin{gathered}
\frac{4}{5}|V(\sigma, t, z)| \leq|V(s, t, z)| \leq \frac{6}{5}|V(\sigma, t, z)|, \\
\frac{3}{5}|V(\sigma, t, z)-V(\sigma)| \leq|V(s, t, z)-V(s)| \leq \frac{7}{5}|V(\sigma, t, z)-V(\sigma)| .
\end{gathered}
$$

Define

$$
d(s):=X(s, t, z)-X(s), s \in[t-\Delta, t],
$$

choose $\bar{s} \in[t-\Delta, t]$ such that $|d(\bar{s})|=\min \{|d(s)| \mid s \in[t-\Delta, t]\}$ and define

$$
\bar{d}(s):=d(\bar{s})+\dot{d}(\bar{s})(s-\bar{s}), s \in[t-\Delta, t] .
$$

Since $d(\bar{s}) \cdot \dot{d}(\bar{s})(s-\bar{s}) \geq 0$ for $s \in[t-\Delta, t]$ we see that

$$
|\bar{d}(s)| \geq|\dot{d}(\bar{s})||s-\bar{s}|, \quad s \in[t-\Delta, t] .
$$


On the other hand

$$
\begin{aligned}
|\ddot{d}(s)-\ddot{\bar{d}}(s)| \leq & \frac{2 \dot{a}(0)}{a^{2}(s)}|V(s, t, z)-V(s)| \\
& +\frac{1}{a^{2}(s)}\left|\partial_{x} W(s, X(s, t, z))-\partial_{x} W(s, X(s))\right| \\
\leq & 2 C_{2} \frac{P(s)^{4 / 3}}{a(s)}
\end{aligned}
$$

and thus

$$
|d(s)-\bar{d}(s)| \leq C_{2} \frac{P(t)^{4 / 3}}{a(t-\Delta)}|s-\bar{s}| \Delta \leq \frac{1}{5} \frac{p}{a(t-\Delta)}|s-\bar{s}| .
$$

By definition of the set $M_{3}$ and Eqn. (6.6)

$$
p \leq \frac{5}{3} a(\bar{s})|\dot{d}(\bar{s})|
$$

so that

$$
|d(s)-\bar{d}(s)| \leq \frac{2}{3}|\dot{d}(\bar{s})||s-\bar{s}|, s \in[t-\Delta, t] .
$$

Together with Eqn. (6.7) this yields

$$
|d(s)| \geq \frac{1}{3}|\dot{d}(\bar{s})||s-\bar{s}|, s \in[t-\Delta, t] .
$$

For $s \in J(z)$ the definition of the set $M_{3}$ and the estimates (6.5) and (6.6) imply that

$$
|d(s)| \geq C r \min \{|v|,|v-V(t)|\}^{-3} .
$$

Combining this estimate with (6.8) and

$$
|\dot{d}(\bar{s})|=\frac{1}{a(\bar{s})}|V(\bar{s}, t, z)-V(\bar{s})| \geq \frac{C}{a(\bar{s})}|v-V(t)|
$$

yields

$$
|d(s)| \geq C \max \left\{\frac{1}{a(t)}|v-V(t)||s-\bar{s}|, \frac{r}{|v|^{3}}, \frac{r}{|v-V(t)|^{3}}\right\}
$$

for $s \in[t-\Delta, t]$. Inserting this estimate in the time integral in (6.4) and distinguishing the cases $|v| \leq|v-V(t)|$ and $|v| \geq|v-V(t)|$ finally yields the estimate

$$
\int_{J(z)} a^{2}(s)|X(s, t, z)-X(s)|^{-2} d s \leq C a^{3}(t) r^{-1}|v|^{2} .
$$


Thus by Lemma 5.2

$$
\begin{aligned}
J & \leq C r^{-1} a^{3}(t) \int_{(X(t)+2 \hat{Q}) \times \mathbb{R}^{3}} v^{2} f(t, z) d z \\
& \leq C r^{-1} a^{3}(t) \int_{S} v^{2} f(t, z) d z \\
& \leq C r^{-1} .
\end{aligned}
$$

Combining the estimates for $I_{1}, I_{2}$, and $I_{3}$ we obtain

$$
\begin{aligned}
\mid a(t) V(t) & -a(t-\Delta) V(t-\Delta) \mid \\
& \leq C\left(1+a(t) p^{4 / 3}+a^{2}(t) r \ln \frac{2 P(t)}{p}+r^{-1} \Delta^{-1}\right) \Delta
\end{aligned}
$$

for

$$
\Delta=\Delta(t)=\min \left\{t, \frac{1}{2 \dot{a}(0)}, \frac{1}{4 P(t)}, \frac{p}{5 C_{2} P(t)^{4 / 3}}\right\}
$$

Now choose $r=a^{-1}(t) P(t)^{1 / 2} \ln ^{-1 / 2}(2 P(t) / p)$ and $p=P(t)^{1 / 3}$. Then for $t$ close enough to $T$, say $t \geq t^{*}$, we have $\Delta(t)=\frac{1}{5 C_{2} P(t)}$ and

$$
|a(t) V(t)-a(t-\Delta) V(t-\Delta)| \leq C a(t) P(t)^{1 / 2+\epsilon} \Delta(t)
$$

for any $\epsilon>0$. Defining $t_{0}:=t$ and $t_{i+1}:=t_{i}-\Delta\left(t_{i}\right)$ as long as $t_{i}>t^{*}$ and observing that $\Delta\left(t_{i}\right) \geq \Delta\left(t_{0}\right)$ we see that there exists $k \in \mathbb{N}$ such that $t_{k+1}<$ $t^{*} \leq t_{k}$. Thus

$$
\begin{aligned}
|a(t) V(t)| & \leq a\left(t^{*}\right)\left|V\left(t^{*}\right)\right|+C a(t) P(t)^{1 / 2+\epsilon} \sum_{i=0}^{k} \Delta\left(t_{i}\right) \\
& \leq C a(t)(1+t) P(t)^{1 / 2+\epsilon}, t \in\left[t^{*}, T[.\right.
\end{aligned}
$$

We conclude that

$$
P(t) \leq C(1+t) P(t)^{1 / 2+\epsilon}, t \in[0, T[,
$$

which proves the asymptotic estimate for $P(t)$ and by Thm. 4.5 shows that $T=\infty$.

Let us now consider Case B: Assume that $T<T_{a}$. Then $c_{1} \leq a(t) \leq c_{2}$ and $|\dot{a}(t)| \leq c_{3}$ for all $t \in\left[0, T\right.$ [ with positive constants $c_{1}, c_{2}, c_{3}$. Now using exactly the same estimates as above we see that

$$
|a(t) V(t)-a(t-\Delta) V(t-\Delta)| \leq C P(t)^{1 / 2+\epsilon} \Delta(t)
$$

for $t$ close enough to $T$ and $\Delta(t):=\frac{1}{C P(t)}$. This yields the same estimate for $P(t)$ on the interval $[0, T[$ as in Case A, in contradiction to Thm. 4.5. Thus, $T=T_{a}$ also in Case $\mathrm{B}$, and the proof is complete. 


\section{References}

[1] Batt, J.: Global symmetric solutions of the initial value problem in stellar dynamics. J. Differential Eqns. 25, 342-364 (1977)

[2] Batt, J., Rein, G.: A rigorous stability result for the Vlasov-Poisson system in three dimensions. To appear in Anal. di Mat. Pura ed Appl.

[3] Batt, J., Rein, G.: Global classical solutions of the periodic VlasovPoisson system in three dimensions. C. R. Acad. Sc. Paris t 313, 411416 (1991)

[4] Brauer, U.: An existence theorem for perturbed Newtonian cosmological models. J. Math. Phys. 33, 1224-1232 (1992)

[5] Glassey, R. T., Schaeffer, J.: On symmetric solutions of the relativistic Vlasov-Poisson system. Commun. Math. Phys. 101, 459-473 (1985)

[6] Horst, E.: On the classical solutions of the initial value problem for the unmodified non-linear Vlasov equation II. Math. Meth. in the Appl. Sci. 4, 19-32 (1982)

[7] Horst, E.: On the asymptotic growth of the solutions of the VlasovPoisson system. Math. Meth. in the Appl. Sci. 16, 75-85 (1993)

[8] Lions, P.-L., Perthame, B.: Propagation of moments and regularity for the 3-dimensional Vlasov-Poisson system. Invent. math. 105, 415-430 (1991)

[9] Peebles, P. J. E.: The Large Scale Structure of the Universe. Princeton University Press, Princeton, 1980

[10] Pfaffelmoser, K.: Global classical solutions of the Vlasov-Poisson system in three dimensions for general initial data. J. Diff. Eqns. 95, 281$303(1992)$

[11] Rein, G.: Generic global solutions of the relativistic Vlasov-Maxwell system of plasma physics. Commun. Math. Phys. 135, 41-78 (1990)

[12] Rein, G., Rendall, A. D.: Global existence of solutions of the spherically symmetric Vlasov-Einstein system with small initial data. Commun. Math. Phys. 150, 561-583 (1992) 
[13] Schaeffer, J.: Global existence of smooth solutions to the VlasovPoisson system in three dimensions. Commun. Part. Diff. Eqns. 16, 1313-1335 (1991)

[14] Schaeffer, J.: Private communication. 